\title{
Editorial
}

\section{Strengthening human resource information system at the Ministry of Health \& Family Welfare of Bangladesh}

\author{
Nuruzzaman M
}

\section{Background}

Human resource is considered to be the leading building block of the health system. Performance of the health system is immensely dependent on how successfully this human resource (HR) is planned, managed and retained. HR management function encompasses formulation and implementation of HR policy, strategies and plan. In order to formulate effective and efficient policy, a reliable and robust knowledgebase is necessary. Building of such knowledge base requires availing $\mathrm{HR}$ data and information such as age, sex, academic qualifications, specialty, length of experience, workplace etc. These are essential information which leads to the development of a human resource management information system (HRIS). Given the importance of HRIS, the World Health Organization (WHO) and Global health Workforce Alliance (Kampala Declaration, 2008) has urged its member states to create their own HRIS.

Realizing the importance of HRIS, the strategic plan on Health, Population and Nutrition Sector Development Programme (HPNSDP, 2011-2016) of the Ministry of Health and Family Welfare (MOHFW) put human resources issues high on the agenda. One of the activities under HRM in the HPNSDP 2011-2016 is to establish a comprehensive HRIS. The MOHFW has been making efforts to rollout this system across the country for better $\mathrm{HRH}$ planning and management for some times but has failed to take off hitherto.

Available information on health care workers is scattered among the Directorate General of Health Services (DGHS), Directorate General of Family Planning (DGFP), Directorate of Nursing Services (DNS), Registrars of regulatory bodies, professional associations and the information is very limited for the purposes of proper planning and decision making. Current $\mathrm{HRH}$

\footnotetext{
* Md Nuruzzaman

HRH Technical Assistant of WHO

HRM Unit, Ministry of Health \& Family Welfare

Email: zaman.sph@gmail.com

*For correspondence
}

information is associated with difficulties in coordination among DGHS, DGFP and DNS in terms of reliability, consistency and accuracy. Additional challenges include lack of HRH data of the private sector, limited capacity of HR data analysis for HR demands, projection and forecast. Further, the existing human resource for health and Family welfare information systems used by the different directorates are not well established, and there is lack of similarity in the forms for updating information and a comprehensive and reliable system for tracking information.

\section{Rationale}

There is need to develop a functioning HRIS, consolidate, harmonize and close the current data gaps for improvements in HRH planning, management, development and decision making. Further, the resulting information can be used to identify priorities and develop strategies to provide the necessary coherency between $\mathrm{HRH}$ and other inputs in the health system including rationalization of public and private partnerships in $\mathrm{HRH}$ at the district, divisional, or national level. Managers and health planners need information about the size, composition, skill sets, training needs, training schools, programs and their capacity, distribution and performance of the public health workforce in order to make informed, well-timed decision.

\section{General objective}

The MOHFW intends to develop a comprehensive computerized HR information system that cover both the public and private health sectors so as to assist managers to plan future HRH needs and manage human resources that are available efficiently and effectively based on information which is reliable, timely, convenient to use and provided at a reasonable cost. Computerized human resources information systems (HRIS) enable health systems to collect, maintain, and analyze health workforce data enabling policy-makers to use reliable data to make informed decisions about the health workforce.

\section{Specific objectives}

The computerized HRIS benefits will include:

- Assisting the tracking of training information of health workers throughout Bangladesh, from pre-service training through licensure 
- Provision of information to facilitate HRH projections, estimating the HRH gap between the current position and projected gap as well as developing HRH plans, strategies and policies. Health workforce planning is important in ensuring that the recruitment, training and deployment of health workers are conducted in the most efficient way possible

- Provision of information for the determination of future staff development and training needs;

Providing assistance in the assessment of the 'health' of MOHFW measured by attitude surveys and turnover and absence statistics leading to the development of motivation, retention and absence control strategies

- To be the basis for analysis of productivity levels and development of productivity improvement programs;

Provision of accurate and relevant information for $\mathrm{HRH}$ management decisions as regards staff deployment, recruitment, migration of health workers, resignation, deputation, retirements, disciplinary action, thus facilitating the development of an integrated and coherent approach to human resources management and planning

- Provision information to allow for the systematic monitoring and evaluation of the Human Resources Management and Development processes within MOHFW and the private sector

- Reduction in the workload of the HRM Unit, eliminating low value tasks while still enabling the function to provide efficient personnel administrative services

\section{Way forward}

To develop a comprehensive HRIS that nurture ownership and leadership needed for establishment and roll out and sustainability, the following steps can be followed:

- Set up an HRIS development steering committee;

- Conduct a situational analysis of the current situation of human resource information system in the both public and private sector

- Define the information needs;

- Specify data requirements

- Specify how the data is to be obtained;

- Determining where the data is to be stored

- Determining how the data is to be held (choice of database)

- Determining who is to be responsible for the system overall and at all levels of the health system including mechanisms for coordinating the system

- Preparation of an implementation plan including piloting

- Designing procedures to operate with the system;

- Training staff in the new procedures

- Monitoring and revising the system after implementation 